\title{
GENERALIZED MINIMUM ABERRATION FOR ASYMMETRICAL FRACTIONAL FACTORIAL DESIGNS ${ }^{1,2}$
}

\author{
By HoNgQUan Xu AND C. F. J. WU \\ University of Michigan
}

\begin{abstract}
By studying treatment contrasts and ANOVA models, we propose a generalized minimum aberration criterion for comparing asymmetrical fractional factorial designs. The criterion is independent of the choice of treatment contrasts and thus model-free. It works for symmetrical and asymmetrical designs, regular and nonregular designs. In particular, it reduces to the minimum aberration criterion for regular designs and the minimum $G_{2}$-aberration criterion for two-level nonregular designs. In addition, by exploring the connection between factorial design theory and coding theory, we develop a complementary design theory for general symmetrical designs, which covers many existing results as special cases.
\end{abstract}

1. Introduction. A fundamental and practically important question for factorial designs is the issue of optimal factor assignment to columns of the design matrix. For regular designs, the minimum aberration criterion proposed by Fries and Hunter (1980) has become the standard criterion for optimal factor assignment. It includes the maximum resolution criterion as a special case and has the advantage that it can rank-order any two designs. In the last 10-15 years research activities on minimum aberration designs have taken off. See, among others, Chen and Wu (1991), Chen (1992), Chen, Sun and Wu (1993), Tang and Wu (1996), Chen and Hedayat (1996), Suen, Chen and Wu (1997), Cheng, Steinberg and Sun (1999) and Cheng and Mukerjee (1998). Because this criterion is based on the defining contrast subgroup, it has no obvious extension to nonregular designs. Tang and Deng (1999) recently proposed a minimum $G_{2}$-aberration criterion (and related criteria) to compare nonregular two-level designs. This criterion has no obvious extension for designs with more than two levels. Therefore, it is of theoretical and practical interest to develop a general criterion for nonregular designs.

The classification of an $s^{n-m}$ design with $s>2$ needs some discussion. For example, consider a regular $3^{3-1}$ design with the defining relation $C=A B$. As a regular design, it is understood that any two factorial effects are either orthogonal or fully aliased. In particular, the interaction $A B$ is fully aliased with the main effect $C$. However, if all factors are quantitative, the linear and quadratic contrasts are often used in analysis. Then the linear-by-linear

Received May 2000; revised January 2001.

${ }^{1}$ Supported by NSA Grant MSPR-00G-091.

2This paper originally appeared in The Annals of Statistics (2001) 29 549-560. Due to typesetting error the cross references did not appear correctly.

AMS 2000 subject classifications. Primary 62K15; secondary 62K05.

Key words and phrases. ANOVA, distance distribution, MacWilliams transforms, minimum aberration, orthogonal arrays, wordlength pattern. 
and linear-by-quadratic interactions of $A$ and $B$ are partially aliased with the main effects of $C$ and are thus estimable in addition to the main effects of $A$, $B$ and $C$. This new point of view was given in Wu and Hamada [(2000), Section 5.6], who also wrote (in Section 7.2) that a $3^{n-m}$ design should be treated as a nonregular design if the linear and quadratic contrasts are used. This example raises another issue, that is, whether the minimum aberration criterion is still meaningful when the linear and quadratic contrasts or orthogonal polynomial contrasts in general are used.

The purpose of this paper is to present an efficient and systematic method for comparing and selecting general fractional factorial designs. With the previous questions in mind, we study treatment contrasts and ANOVA models for general asymmetrical fractional factorial designs in Section 2. In Section 3 we propose a generalized minimum aberration criterion and provide a statistical justification. The connection between factorial design theory and coding theory is explored and utilized to establish the relationship between the new criterion and the minimum aberration in Section 4 and to develop a complementary design theory for general symmetrical designs in Section 5. Finally the proposed criterion is applied to select designs for the commonly used 18-run orthogonal arrays in Section 6.

The rest of this section is devoted to notation and definitions. Let $w t(u)$ be the number of nonzero elements of a vector $u=\left(u_{1}, \ldots, u_{n}\right), \mathbb{Z}_{s}=\{0,1, \ldots, s-$ 1 \} be the integer ring with modulus $s,|S|$ be the number of elements of a set $S$, $\|C\|^{2}=\sum_{i, j}\left|c_{i j}\right|^{2}$ be the squared norm of a matrix $C=\left(c_{i j}\right),\langle u, x\rangle=\sum u_{j} x_{j}$ be the inner product of two vectors $u=\left(u_{1}, \ldots, u_{n}\right)$ and $x=\left(x_{1}, \ldots, x_{n}\right)$, and $\delta_{u, x}$ be the Kronecker delta, which equals 1 if $u=x$ and 0 otherwise.

An asymmetrical (or mixed-level) design of $N$ runs, $n$ factors and with levels $s_{1}, \ldots, s_{n}$ is denoted by $\left(N, s_{1} \cdots s_{n}\right)$. An $\left(N, s_{1} \cdots s_{n}\right)$-design is a set of $N$ row vectors or an $N \times n$ matrix in which each row represents a run and each column represents a factor. The $j$ th column takes values from a set of $s_{j}$ symbols, say, $\left\{0,1, \ldots, s_{j}-1\right\}$. For example, an $\left(N, s_{1}^{n_{1}} s_{2}^{n_{2}}\right)$-design has $n_{1}$ factors of $s_{1}$ levels and $n_{2}$ factors of $s_{2}$ levels. In particular, an $\left(N, s^{n}\right)$-design is symmetrical. Two designs are isomorphic if one can be obtained from the other through permutations of rows, columns and symbols in each column.

An asymmetrical (or mixed-level) orthogonal array of $N$ runs, $n$ factors, strength $t$ and with levels $s_{1}, \ldots, s_{n}$, denoted by $O A\left(N, s_{1} \cdots s_{n}, t\right)$, is an $\left(N, s_{1} \cdots s_{n}\right)$-design in which all possible level combinations for any $t$ factors appear equally often.

For a prime power $s$, a regular $s^{n-m}$ fractional factorial design $D$ is an $\left(s^{n-m}, s^{n}\right)$-design that is defined by some defining words. All the defining words form a group, called the defining contrast subgroup. Let $A_{j}(D)$ be the number of degrees of freedom carried by distinct defining words of length $j$. We call the vector $\left(A_{1}(D), \ldots, A_{n}(D)\right)$ the wordlength pattern. Note that the $A_{j}(D)$ according to our definition are $s-1$ times the $A_{j}(D)$ as defined in the traditional literature in the regular case; vide Suen, Chen and Wu (1997). This change helps in introducing our generalized minimum aberration while at the same time it has no effect on the definitions of resolution or minimum aberra- 
tion in the regular case. The resolution is the smallest $j$ such that $A_{j}(D)>0$. For two regular $s^{n-m}$ designs $D_{1}$ and $D_{2}, D_{1}$ is said to have less aberration than $D_{2}$ if there exists an $r, 1 \leq r \leq n$, such that $A_{r}\left(D_{1}\right)<A_{r}\left(D_{2}\right)$ and $A_{j}\left(D_{1}\right)=A_{j}\left(D_{2}\right)$ for $j=1, \ldots, r-1$. $D_{1}$ is said to have minimum aberration if there is no other regular design with less aberration than $D_{1}$. In short, the minimum aberration criterion is to sequentially minimize $A_{j}(D)$ for $j=1, \ldots, n$.

For an $\left(N, 2^{n}\right)$-design $D$, define

$$
A_{j}(D)=N^{-2} \sum_{w t(u)=j}\left|J_{u}(D)\right|^{2} \quad \text { for } j=1, \ldots, n,
$$

where $J_{u}(D)=\sum_{x \in D}(-1)^{\langle u, x\rangle}$ and the summation is over all $n$-tuple binary vectors [i.e., $(0,1)$-vectors] $u$ with $j$ nonzero elements. For $u=\left(u_{1}, \ldots, u_{n}\right)$, $J_{u}(D)$ does not depend on the $k$ th column of $D$ if $u_{k}=0$. The minimum $G_{2}$-aberration criterion proposed by Tang and Deng (1999) is to sequentially minimize $A_{j}(D)$ in (1) for $j=1, \ldots, n$. It reduces to minimum aberration for two-level regular designs.

2. Treatment contrasts and ANOVA models. Let $G_{i}=\left\{0,1, \ldots, s_{i}-\right.$ 1\} and $H=G_{1} \times \cdots \times G_{n}$. For a full factorial $s_{1} \times \cdots \times s_{n}$ experiment in which the $i$ th factor has $s_{i}$ levels, consider the following general ANOVA model

$$
E(Y(x))=\sum_{u \in H} \chi_{u}(x) \beta_{u},
$$

where $Y(x)$ is the response of treatment combination $x \in H, \beta_{u}$ are treatment contrasts (or factorial effects) and $\chi_{u}(x)$ are contrast coefficients. For $u \in H$ with $w t(u)=i, \beta_{u}$ is an $i$-factor interactions. In particular, $\beta_{0}=\beta_{(0, \ldots, 0)}$ is the general mean which has coefficients 1 , that is, $\chi_{0}(x)=\chi_{(0, \ldots, 0)}(x)=1$ for any $x \in H$. For convenience, the contrasts are said to be orthonormal if

$$
\sum_{x \in H} \chi_{u}(x) \overline{\chi_{v}(x)}=|H| \delta_{u, v} \quad \text { for any } u, v \in H,
$$

where $\overline{\chi_{v}(\cdot)}$ is the complex conjugate of $\chi_{v}(\cdot)$.

In this paper, as often done in practice, we only consider contrasts defined by tensor products:

$$
\begin{aligned}
\chi_{u}(x)= & \prod_{i=1}^{n} \chi_{u_{i}}^{\left(s_{i}\right)}\left(x_{i}\right) \\
& \quad \text { for } u=\left(u_{1}, \ldots, u_{n}\right) \in H \text { and } x=\left(x_{1}, \ldots, x_{n}\right) \in H,
\end{aligned}
$$

where $\left\{\chi_{u_{i}}^{\left(s_{i}\right)}, u_{i} \in G_{i}\right\}$ are contrasts for the $i$ th factor which has $s_{i}$ levels. Then, condition (3) is equivalent to the condition that for all $i,\left\{\chi_{u_{i}}^{\left(s_{i}\right)}, u_{i} \in G_{i}\right\}$ are orthonormal, that is, $\sum_{x_{i} \in G_{i}} \chi_{u_{i}}^{\left(s_{i}\right)}\left(x_{i}\right) \overline{\chi_{v_{i}}^{\left(s_{i}\right)}\left(x_{i}\right)}=\left|G_{i}\right| \delta_{u_{i}, v_{i}}$ for any $u_{i}, v_{i} \in G_{i}$.

Commonly used contrasts are from orthogonal polynomials, especially for quantitative factors. Another class is the complex contrasts proposed by Bailey (1982). The complex contrasts are of no meaning in practice but of great use in theory. For an $s$-level factor, the complex contrasts are defined to be $\chi_{u}^{(s)}(x)=$ 
$\xi^{u \cdot x}$ for $u, x \in \mathbb{Z}_{s}$, where $\xi$ is a primitive $s$ th root of unity in $\mathbb{C}$, say $\xi=e^{2 \pi i / s}$. The original work of Bailey requires $s$ to be a prime which is unnecessary. For simplicity, we use $i$ for both $\sqrt{-1}$ and an index. The meaning of $i$ should be clear from the context.

The ANOVA model (2) is not unique because orthonormal contrasts are not unique when some factors have more than two levels. As a consequence, it is of little meaning to consider one particular model for a general factorial design. We need some invariant measure to assess the goodness of a design. In the next section we shall introduce the concept of a generalized minimum aberration to tackle this problem.

3. Generalized minimum aberration and its statistical justification. For an $\left(N, s_{1} \cdots s_{n}\right)$-design $D$, it is convenient to rewrite the ANOVA model (2) in the following form

$$
Y=X_{0} \alpha_{0}+X_{1} \alpha_{1}+\cdots+X_{n} \alpha_{n}+\varepsilon
$$

where $Y$ is the vector of $N$ observations, $\alpha_{j}$ is the vector of all $j$-factor interactions and $X_{j}$ is the matrix of contrast coefficients for $\alpha_{j}$. Specifically, $Y=(Y(x))_{x \in D}, \alpha_{j}=\left(\beta_{u}\right)_{w t(u)=j}$ and $X_{j}=\left[\chi_{u}(x)\right]_{x \in D, w t(u)=j}$. Clearly, $X_{0}$ is the vector of ones. It is important to note that $\left\|\bar{X}_{i}^{T} X_{j}\right\|^{2}$ is independent of the choice of orthonormal contrasts [see Remark 2.3.1 of Dey and Mukerjee (1999)].

For given orthonormal contrasts $\left\{\chi_{u}, u \in H\right\}$ satisfying (3) and (4), define

$$
A_{j}(D)=N^{-2}\left\|\bar{X}_{0}^{T} X_{j}\right\|^{2}=N^{-2} \sum_{w t(u)=j}\left|\chi_{u}(D)\right|^{2} \quad \text { for } j=0, \ldots, n,
$$

where $\chi_{u}(D)=\sum_{x \in D} \chi_{u}(x)$ and the summation is over all $u \in H$ with $j$ nonzero elements. Clearly $A_{0}(D)=1$. The value $A_{j}(D)$ measures the overall aliasing between all $j$-factor interactions and the general mean. For convenience, the vector $\left(A_{1}(D), \ldots, A_{n}(D)\right)$ is called the generalized wordlength pattern. Then the generalized minimum aberration (GMA) criterion is to sequentially minimize $A_{j}(D)$ in (6) for $j=1, \ldots, n$. It is independent of the choice of orthonormal contrasts and thus model-free. Furthermore, isomorphic designs are equivalent under GMA because they have the same generalized wordlength pattern.

An underlying assumption for minimum aberration and GMA is the hierarchical ordering principle [Wu and Hamada (2000), Section 3.5]: (i) lower order effects are more likely to be important than higher order effects, (ii) effects of the same order are equally likely to be important.

It is evident that minimum $G_{2}$-aberration is a special case of GMA. As will be shown in the next section, the generalized wordlength pattern is the same as the wordlength pattern for a regular design and thus GMA reduces to minimum aberration for regular designs.

The GMA introduced here is different from the minimum aberration proposed by $\mathrm{Wu}$ and Zhang (1993) for $4^{m} 2^{n}$ designs because the former obeys 
the hierarchical ordering principle while the latter does not. Wu and Zhang argued that interactions between four-level and two-level factors are less important than interactions between two two-level factors, which violates the second part of the hierarchical principle.

Tang and Deng (1999) provided a statistical justification for minimum $G_{2^{-}}$ aberration. Here we extend their approach for GMA. Suppose the main effects are of primary interest and a main-effect model is fitted, that is, assuming $\alpha_{j}=0$ in (5) for $j \geq 2$. Then an unbiased estimate of the main effects $\alpha_{1}$ is $\hat{\alpha}_{1}=\left(\bar{X}_{1}^{T} X_{1}\right)^{-1} \bar{X}_{1}^{T} Y=N^{-1} \bar{X}_{1}^{T} Y$ if $D$ is an orthogonal array of strength 2 . However, under the full model (5),

$$
E\left(\hat{\alpha}_{1}\right)=\alpha_{1}+C_{2} \alpha_{2}+\cdots+C_{n} \alpha_{n},
$$

where $C_{j}=N^{-1} \bar{X}_{1}^{T} X_{j}$, which depend on $D$, are the aliasing matrices. The value $\left\|C_{j}\right\|^{2}$, which is independent of the choice of orthonormal contrasts, measures the contamination of non-negligible $j$-factor interactions on the estimation of main effects. In the spirit of the hierarchical ordering principle, a good design should sequentially minimize $\left\|C_{j}\right\|^{2}$ for $j=2, \ldots, n$. The following result on the relationship between $A_{i}$ and $\left\|C_{j}\right\|^{2}$ is important in this regard.

LEMMA 1. For a symmetrical $\left(N, s^{n}\right)$-design,

$\left\|C_{j}\right\|^{2}=(j+1) A_{j+1}+j(s-2) A_{j}+(n-j+1)(s-1) A_{j-1} \quad$ for $j=2, \ldots, n$.

For an asymmetrical $O A\left(N, s_{1} \cdots s_{n}, t\right)$,

$$
\left\|C_{j}\right\|^{2}=(j+1) A_{j+1} \quad \text { for } j=2, \ldots, t .
$$

The proof is given in the Appendix. Because $A_{1}=A_{2}=0$ if $D$ is an orthogonal array of strength 2 (see Theorem 4 below), Lemma 1 shows that sequentially minimizing $A_{3}, A_{4}, \ldots$ is equivalent to sequentially minimizing $\left\|C_{2}\right\|^{2},\left\|C_{3}\right\|^{2}, \ldots$. This leads to the following theorem, which generalizes Tang and Deng's (1999) result.

THEOREM 1. The generalized minimum aberration criterion sequentially minimizes the contamination of non-negligible $j$-factor interactions on the estimation of main effects for $j=2, \ldots, e$, where e equals the number of factors for symmetrical designs and the strength for asymmetrical designs.

Note that the computation is cumbersome according to the definition (6). In the next section we shall derive an alternative and simpler expression for $A_{j}(D)$ in (6) which exploits the connection between factorial design theory and coding theory.

4. Connection with coding theory. This section studies the connection between factorial design theory and coding theory. The relationship between regular designs and linear codes was first observed by Bose (1961). To study 
the general relationship we need some recently developed results in coding theory which can be found in MacWilliams and Sloane (1977), Roman (1992) and van Lint (1999). For clarity, we shall consider symmetrical and asymmetrical designs separately.

First consider symmetrical designs. An $\left(N, s^{n}\right)$-design $D$ is a set of $N$ row vectors of length $n$. For two vectors $a$ and $b$, the Hamming distance $d_{H}(a, b)$ is the number of places where they differ. The distance distribution of $D$ is $\left(B_{0}(D), B_{1}(D), \ldots, B_{n}(D)\right)$, where

$$
B_{j}(D)=N^{-1}\left|\left\{(a, b): d_{H}(a, b)=j, a \in D, b \in D\right\}\right| \quad \text { for } j=0, \ldots, n .
$$

The MacWilliams transforms of the distance distribution are defined as

$$
B_{j}^{\prime}(D)=N^{-1} \sum_{i=0}^{n} B_{i}(D) P_{j}(i ; n, s) \quad \text { for } j=0, \ldots, n,
$$

where $P_{j}(x ; n, s)=\sum_{i=0}^{j}(-1)^{i}(s-1)^{j-i}\left(\begin{array}{c}x \\ i\end{array}\right)\left(\begin{array}{c}n-x \\ j-i\end{array}\right)$ are the Krawtchouk polynomials. By the orthogonality of the Krawtchouk polynomials, it is easy to show that

$$
B_{j}(D)=N s^{-n} \sum_{i=0}^{n} B_{i}^{\prime}(D) P_{j}(i ; n, s) \quad \text { for } j=0, \ldots, n .
$$

The equations (7) and (8) are known as the generalized MacWilliams identities.

The connection is established through complex contrasts which are known as characters in coding theory. For an $\left(N, s^{n}\right)$-design, the complex contrasts are $\chi_{u}(x)=\xi^{\langle u, x\rangle}$ for $u, x \in \mathbb{Z}_{s}^{n}$, where $\xi=e^{2 \pi i / s}$. The Delsarte theory shows that

$$
B_{j}^{\prime}(D)=N^{-2} \sum_{w t(u)=j}\left|\chi_{u}(D)\right|^{2} \quad \text { for } j=0, \ldots, n,
$$

where $u \in \mathbb{Z}_{s}^{n}$. By comparing $B_{j}^{\prime}(D)$ with the definition of $A_{j}(D)$ in (6), we obtain the following result.

THEOREM 2. For an $\left(N, s^{n}\right)$-design $D$, the generalized wordlength pattern is the MacWilliams transform of the distance distribution, that is, $A_{j}(D)=$ $B_{j}^{\prime}(D)$ for $j=0,1, \ldots, n$.

It is well known that a regular design is a linear code and its defining contrast subgroup is the dual code. For linear codes, the distance distribution is the same as the weight distribution. The wordlength pattern of a regular design $D$ is the weight distribution of its dual code, which is the MacWilliams transform of the weight distribution of $D$. Thus, we have the following result.

THEOREM 3. For a regular design the generalized wordlength pattern is the same as the wordlength pattern and hence generalized minimum aberration reduces to minimum aberration for regular designs. 
It is worth noting that regular minimum aberration designs may not have GMA when nonregular designs are also considered. For example, consider $\left(128,2^{15}\right)$-designs. There is an $O A\left(128,2^{15}, 4\right)$ which is nonregular [see $\mathrm{He}$ dayat, Sloane and Stufken (1999)]; however, a regular minimum aberration design only has strength 3 .

Next consider asymmetrical designs. For simplicity, consider an $\left(N, s_{1}^{n_{1}} s_{2}^{n_{2}}\right)$ design $D$. The extension to general asymmetrical designs is obvious. It is convenient to split a row vector into two parts, for example, $a=\left(a_{1}, a_{2}\right)$, where $a_{i} \in \mathbb{Z}_{s_{i}}^{n_{i}}$. For $0 \leq j_{1} \leq n_{1}, 0 \leq j_{2} \leq n_{2}$, define

$$
\begin{array}{r}
B_{j_{1}, j_{2}}(D)=N^{-1} \mid\left\{(a, b): d_{H}\left(a_{1}, b_{1}\right)=j_{1}, d_{H}\left(a_{2}, b_{2}\right)=j_{2},\right. \\
\left.a=\left(a_{1}, a_{2}\right) \in D, b=\left(b_{1}, b_{2}\right) \in D\right\} \mid
\end{array}
$$

and

$$
B_{j_{1}, j_{2}}^{\prime}(D)=N^{-1} \sum_{i_{1}=0}^{n_{1}} \sum_{i_{2}=0}^{n_{2}} B_{i_{1}, i_{2}}(D) P_{j_{1}}\left(i_{1} ; n_{1}, s_{1}\right) P_{j_{2}}\left(i_{2} ; n_{2}, s_{2}\right) .
$$

Again the connection is established through complex contrasts. In this case, the complex contrasts are $\chi_{u}(x)=\chi_{u_{1}}\left(x_{1}\right) \chi_{u_{2}}\left(x_{2}\right)=\xi_{1}^{\left\langle u_{1}, x_{1}\right\rangle} \xi_{2}^{\left\langle u_{2}, x_{2}\right\rangle}$ for $u=$ $\left(u_{1}, u_{2}\right), x=\left(x_{1}, x_{2}\right) \in \mathbb{Z}_{s_{1}}^{n_{1}} \times \mathbb{Z}_{s_{2}}^{n_{2}}$, where $\xi_{1}=e^{2 \pi i / s_{1}}$ and $\xi_{2}=e^{2 \pi i / s_{2}}$. Sloane and Stufken (1996) showed that

$$
B_{j_{1}, j_{2}}^{\prime}(D)=N^{-2} \sum_{w t\left(u_{1}\right)=j_{1}} \sum_{w t\left(u_{2}\right)=j_{2}}\left|\chi_{u}(D)\right|^{2},
$$

where $u=\left(u_{1}, u_{2}\right) \in \mathbb{Z}_{s_{1}}^{n_{1}} \times \mathbb{Z}_{s_{2}}^{n_{2}}$. Through these expressions, they showed that $D$ is an orthogonal array of strength $t$ if and only if $B_{j_{1}, j_{2}}^{\prime}(D)=0$ for $1 \leq$ $j_{1}+j_{2} \leq t$. By comparing $B_{j_{1}, j_{2}}^{\prime}(D)$ with the definition of $A_{j}(D)$ in (6), we obtain the following result.

THEOREM $4 . \quad$ (i) For an $\left(N, s_{1}^{n_{1}} s_{2}^{n_{2}}\right)$-design $D, A_{j}(D)=\sum_{j_{1}+j_{2}=j} B_{j_{1}, j_{2}}^{\prime}(D)$ for $0 \leq j \leq n_{1}+n_{2}$;

(ii) $D$ is an orthogonal array of strength $t$ if and only if $A_{j}(D)=0$ for $1 \leq j \leq t$.

In the next two sections, we will use these formulas to develop a general complementary design theory and to compute the generalized wordlength patterns for the study of the commonly used 18-run orthogonal arrays.

5. Complementary designs. There has been increasing interest in the characterization of designs in terms of their complementary designs. The technique is particularly powerful when the number of factors in the complementary design is much smaller than that in the original design. A theory has been developed for two-level regular designs by Tang and Wu (1996), for general regular designs by Suen, Chen and Wu (1997) and for general two-level designs by Tang and Deng (1999). Here we develop a complementary design 
theory for general symmetrical designs, which covers the previous results as special cases.

Suppose $H$ is an $\left(N, s^{p}\right)$-design. Call $(D, \bar{D})$ a pair of complementary designs from $H$ if they are a column partition of $H$. Without loss of generality, let $D$ consist of the first $n$ columns and $\bar{D}$ consist of the remaining $\bar{n}=p-n$ columns. The complementary design problem is to express $\left\{A_{t}(D)\right\}$ in terms of $\left\{A_{t}(\bar{D})\right\}$.

The general theory established here requires that $H$ is a saturated orthogonal array of strength 2 . The saturation imposes a condition on the parameters, i.e., $N-1=(s-1) p$. Nevertheless, this setting is more general than that of Suen, Chen and Wu (1997) and Tang and Deng (1999). The combinatorial approach of Tang and Deng (1999) is complicated even for the two-level case. There seems to be no easy way to extend their approach to the general case. On the other hand, the coding theory approach of Suen, Chen and Wu (1997) is more general and has a clear extension. Here we adopt and extend their approach to the general case. The basic idea is to consider distance distributions rather than weight distributions. With the connection established in the previous section, we are ready to develop a general theory. A key observation about saturated orthogonal arrays is the following lemma, which follows from Lemma 1 of Mukerjee and Wu (1995).

LEMMA 2. The Hamming distance between any two distinct rows of a saturated $O A\left(N, s^{p}, 2\right)$ is $N s^{-1}$.

Throughout this section, $H$ is a saturated $O A\left(N, s^{p}, 2\right)$ with $N-1=$ $(s-1) p, D$ and $\bar{D}$ consist of the first $n$ and respectively remaining $\bar{n}$ columns of $H$ with $n+\bar{n}=p$.

LEMMA 3. The distance distributions $\left\{B_{i}(D)\right\}$ and $\left\{B_{i}^{\prime}(\bar{D})\right\}$ satisfy the following equations:

(i) $B_{i}(D)=0$, for $i>\min \left(N s^{-1}, n\right)$;

(ii) $B_{i}(\bar{D})=0$, for $i>\min \left(N s^{-1}, \bar{n}\right)$;

(iii) $B_{i}(D)=B_{N s^{-1}-i}(\bar{D})+\delta_{0, i}-\delta_{N s^{-1}, i}$, for $i=0, \ldots, N s^{-1}$.

PRoOF. For a pair of row vectors $(a, b)$ of $H=(D, \bar{D})$, let $a=\left(a_{1}, a_{2}\right)$ and $b=\left(b_{1}, b_{2}\right)$ such that $a_{1}, b_{1} \in D$ and $a_{2}, b_{2} \in \bar{D}$. Then $d_{H}(a, b)=$ $d_{H}\left(a_{1}, b_{1}\right)+d_{H}\left(a_{2}, b_{2}\right)$. From Lemma $2, d_{H}(a, b)=N s^{-1}$ if $a$ and $b$ are distinct and $d_{H}(a, b)=0$ otherwise. Then the lemma follows from the definition of the distance distribution.

LEMMA 4. The generalized wordlength patterns $\left\{A_{i}(D)\right\}$ and $\left\{A_{i}(\bar{D})\right\}$ satisfy the following equations:

$$
A_{k}(D)=C_{k}+\sum_{j=0}^{\bar{n}} C_{k j} A_{j}(\bar{D}) \quad \text { for } k=0, \ldots, n
$$


where $C_{k}=N^{-1}\left[P_{k}(0 ; n, s)-P_{k}\left(N s^{-1} ; n, s\right)\right]$ and $C_{k j}=s^{-\bar{n}} \sum_{i=0}^{\bar{n}} P_{k}\left(N s^{-1}-\right.$ $i ; n, s) P_{i}(j ; \bar{n}, s)$ for $j=0, \ldots, \bar{n}$.

Given Theorem 2 and Lemma 3, the proof is analogous to that of Theorem 1 of Suen, Chen and Wu (1997). The next lemma follows from the proofs of Theorem 2 and Corollary 1 of Suen, Chen and Wu (1997).

Lemma 5. The coefficients $C_{k j}$ in Lemma 4 are the following:

(i) $C_{k j}=0$ for $k<j$;

(ii) $C_{k j}=\sum_{t+u+v=k-j}\left(\begin{array}{c}n-N s^{-1} \\ t\end{array}\right)\left(\begin{array}{c}N s^{-1}-\bar{n} \\ u\end{array}\right)\left(\begin{array}{c}\bar{n}-j \\ v\end{array}\right)(-1)^{u+j}(s-2)^{v}(s-1)^{t}$ for $k \geq j$;

(iii) $C_{k, k-1}=(-1)^{k}[1+(s-2)(k-1)]$; and

(iv) $C_{k k}=(-1)^{k}$.

For $s=2, C_{k j}=(-1)^{k-\lfloor(k-j) / 2\rfloor}\left(\begin{array}{c}n-N / 2 \\ \lfloor(k-j) / 2\rfloor\end{array}\right)$ for $k \geq j$, where $\lfloor x\rfloor$ is the largest integer less than or equal to $x$, and $C_{k, k-1}=C_{k k}=(-1)^{k}$.

The next theorem follows from Lemmas 4 and 5 .

THEOREM 5. The generalized wordlength patterns $\left\{A_{i}(D)\right\}$ and $\left\{A_{i}(\bar{D})\right\}$ satisfy the following equations:

$$
\begin{aligned}
A_{k}(D)= & \left(C_{k}+C_{k 0}\right)+\sum_{j=3}^{k-2} C_{k j} A_{j}(\bar{D})+(-1)^{k}[1+(s-2)(k-1)] A_{k-1}(\bar{D}) \\
& +(-1)^{k} A_{k}(\bar{D}),
\end{aligned}
$$

for $k=0, \ldots, n$, where $C_{k j}$ and $C_{k}$ are given in Lemmas 4 and 5 .

Clearly these identities include as special cases the results derived by Tang and Wu (1996), Suen, Chen and Wu (1997) and Tang and Deng (1999). In addition, explicit expressions given in Theorem 5 are not available in Tang and Deng (1999).

From Theorem 5, we have the following general relation:

$$
A_{k}(D)=(-1)^{k} A_{k}(\bar{D})+\text { lower order terms. }
$$

Therefore, the general rules given in Suen, Chen and Wu (1997) still work for nonregular symmetrical designs. However, the situation for nonregular designs is more complicated than that of regular designs, which was also noted by Tang and Deng (1999). For a given run size there are many non-isomorphic saturated orthogonal arrays. For example, Lam and Tonchev (1996) showed that there are 68 non-isomorphic $O A\left(27,3^{13}, 2\right)$ 's, among which only one is regular. It is computationally intensive to enumerate all subdesigns from them. Nevertheless, some general results can still be obtained through the complementary design theory. For instance, all subdesigns of 12 (or 11) columns 
from any of these saturated orthogonal arrays have the same generalized wordlength pattern.

Finally it is of theoretical and practical interest to develop a complementary design theory for asymmetrical designs. It seems feasible to follow the current approach; however, the details are much more complicated.

6. Application: GMA designs from 18-run orthogonal arrays. In this section we apply the GMA to study the commonly used 18-run orthogonal arrays given in Table 7C.2 of Wu and Hamada (2000). First consider an $O A\left(18,3^{7}, 2\right)$ given by columns 2 to 8 in their table. For three factors, there are three non-isomorphic subdesigns according to Wang and Wu (1995). Following their notation, let design 18-3.1 consist of columns $(3,4,5)$, design 18-3.2 of columns $(2,3,8)$, and design $18-3.3$ of columns $(2,4,5)$, respectively. Design 18-3.1 has 17 degrees of freedom for estimating factorial effects while designs 18-3.2 and 18-3.3 have 14 and 8 degrees of freedom, respectively. It is easy to verify that the generalized wordlength patterns (or wordlength patterns for short) are $(0,0,0.5),(0,0,1)$ and $(0,0,2)$ respectively. Hence, design 18-3.1 has less aberration than design 18-3.2, which in turn has less aberration than design 18-3.3. This is consistent with the ordering made in Wang and Wu. For four factors, there are four non-isomorphic subdesigns according to Wang and Wu. Let design 18-4.1 consist of columns $(3,4,5,6)$, design 18-4.2 of columns $(2,3,4,7)$, design $18-4.3$ of columns $(2,3,4,5)$ and design 18-4.4 of columns $(2,3,6,8)$. The wordlength patterns are $(0,0,2,1.5),(0,0,2.5,1),(0,0,3.5,0)$ and $(0,0,3.5,0)$ respectively. Hence, design $18-4.1$ has less aberration than design 18-4.2, which in turn has less aberration than designs 18-4.3 and 18-4.4. Note that designs 18-4.3 and 18-4.4 have the same wordlength pattern though they are not isomorphic. For five factors, the number of non-isomorphic subdesigns is unknown in the literature due to the complexity of verifying design isomorphism. There is no efficient method to verify design isomorphism. Nevertheless, it is easy to verify that there are four subdesigns having different wordlength patterns. The GMA calculations show that any five columns not containing column 2 form a GMA design. For six factors, columns 3 to 8 form a GMA design. In summary, we reach an interesting conclusion that any design not containing column 2 has GMA.

Next consider an $O A\left(18,2^{1} 3^{7}, 2\right)$ given by columns 1 to 8 in Table 7 C.2 of $\mathrm{Wu}$ and Hamada (2000). There is only one choice for the two-level column. The situation is complicated by the presence of the two-level column. For example, the number of non-isomorphic subdesigns with 3 to 7 factors is not known due to the complexity of verifying design isomorphism. However, we can easily calculate the wordlength patterns for all possible subdesigns. There are 2, 6, 5, 5 and 2 different wordlength patterns for $n=3,4,5,6$ and 7 factors, respectively. Table 1 lists a GMA design for each $n$.

Finally consider an $O A\left(18,6^{1} 3^{6}, 2\right)$ given by columns $1^{\prime}$ and 3 to 8 in Table 7C.2 of $\mathrm{Wu}$ and Hamada (2000). There is only one choice for the six-level column. The GMA calculations show that all subdesigns of $n$ factors have the same wordlength pattern for $n=3,4,5,6$. 
TABLE 1

GMA Designs from $O A\left(18,2^{1} 3^{7}, 2\right)$

\begin{tabular}{l|lllllll}
\hline $\boldsymbol{n}$ & \multicolumn{7}{|c}{ Columns } \\
\hline 3 & 1 & 3 & 6 & & & & \\
4 & 1 & 3 & 6 & 7 & & & \\
5 & 1 & 2 & 3 & 6 & 7 & & \\
6 & 1 & 2 & 3 & 4 & 6 & 7 & \\
7 & 1 & 3 & 4 & 5 & 6 & 7 & 8 \\
\hline
\end{tabular}

\section{APPENDIX}

PROOF OF LEMMA 1. We use complex contrasts for the proof because both $\left\|C_{j}\right\|^{2}$ and $A_{j}$ are invariant with respect to the choice of orthonormal contrasts. For an $\left(N, s^{n}\right)$-design $D$, the complex contrasts are $\chi_{u}(x)=\xi^{\langle u, x\rangle}$ for $u, x \in \mathbb{Z}_{s}^{n}$, where $\xi=e^{2 \pi i / s}$. Clearly $\overline{\chi_{u}(x)} \chi_{v}(x)=\chi_{v-u}(x)$. The elements of $\bar{X}_{1}^{T} X_{j}$ are $\sum_{x \in D} \overline{\chi_{u}(x)} \chi_{v}(x)=\sum_{x \in D} \chi_{v-u}(x)=\chi_{v-u}(D)$, where $w t(u)=1$ and $w t(v)=j$. Then

$$
\left\|C_{j}\right\|^{2}=N^{-2}\left\|\bar{X}_{1}^{T} X_{j}\right\|^{2}=N^{-2} \sum_{w t(u)=1} \sum_{w t(v)=j}\left|\chi_{v-u}(D)\right|^{2} .
$$

The summation can be split into three parts according to $w t(v-u)=j+1, j$ or $j-1$. Given any $v-u$ such that $w t(v-u)=j+1$ ( $j$ and $j-1$, respectively), there are $j+1[j(s-2)$ and $(n-j+1)(s-1)$, respectively] possible ways of choosing $u$ and $v$ such that $w t(u)=1$ and $w t(v)=j$. Thus the first statement follows from the definition of $A_{j}$ in (6). With a similar argument, it can be shown that for an $\left(N, s_{1}^{n_{1}} s_{2}^{n_{2}}\right)$-design,

$$
\begin{aligned}
\left\|C_{j}\right\|^{2}= & (j+1) \sum_{j_{1}+j_{2}=j+1} B_{j_{1}, j_{2}}^{\prime}+\sum_{j_{1}+j_{2}=j} B_{j_{1}, j_{2}}^{\prime}\left[j_{1}\left(s_{1}-2\right)+j_{2}\left(s_{2}-2\right)\right] \\
& +\sum_{j_{1}+j_{2}=j-1} B_{j_{1}, j_{2}}^{\prime}\left[\left(n_{1}-j_{1}\right)\left(s_{1}-1\right)+\left(n_{2}-j_{2}\right)\left(s_{2}-1\right)\right] .
\end{aligned}
$$

Then the second statement follows from Theorem 4.

Acknowledgments. The authors are grateful to the referees for their valuable comments.

\section{REFERENCES}

BAILEY, R. A. (1982). The decomposition of treatment degrees of freedom in quantitative factorial experiments. J. Roy. Statist. Soc. Ser. B 44 63-70.

Bose, R. C. (1961). On some connections between the design of experiments and information theory. Bull. Inst. Internat. Statist. 38 257-271. 
Chen, H. and Hedayat, A. S. (1996). $2^{n-l}$ designs with weak minimum aberration. Ann. Statist. $242536-2548$.

CHEN, J. (1992). Some results on $2^{n-k}$ fractional factorial designs and search for minimum aberration designs. Ann. Statist. $202124-2141$.

Chen, J., Sun, D. X. and Wu, C. F. J. (1993). A catalogue of two-level and three-level fractional factorial designs with small runs. Internat. Statist. Rev. 61 131-145.

CHen, J. and WU, C. F. J. (1991). Some results on $s^{n-k}$ fractional factorial designs with minimum aberration or optimal moments. Ann. Statist. 19 1028-1041.

Cheng, C. S. and MukeRJEe, R. (1998). Regular fractional factorial designs with minimum aberration and maximum estimation capacity. Ann. Statist. 26 2289-2300.

Cheng, C. S., Steinberg, D. M. and Sun, D. X. (1999). Minimum aberration and model robustness for two-level fractional factorial designs. J. Roy. Statist. Soc. Ser. B 61 85-93.

Dey, A. and MukerJee, R. (1999). Fractional Factorial Plans. Wiley, New York

FrIES, A. and HunteR, W. G. (1980). Minimum aberration $2^{k-p}$ designs. Technometrics $22601-$ 608.

Hedayat, A. S., Sloane, N. J. A. and Stufken, J. (1999). Orthogonal Arrays: Theory and Applications. Springer, New York.

LAM, C. and Tonchev, V. D. (1996). Classification of affine resolvable 2-(27,9,4) designs. J. Statist. Plann. Inference 56 187-202.

MacWilliams, F. J. and Sloane, N. J. A. (1977). The Theory of Error-correcting Codes. NorthHolland, Amsterdam.

MuKERJEe, R. and WU, C. F. J. (1995). On the existence of saturated and nearly saturated asymmetrical orthogonal arrays. Ann. Statist. 23 2102-2115.

Roman, S. (1992). Coding and Information Theory. Springer, New York.

Sloane, N. J. A. and Stufken, J. (1996). A linear programming bound for orthogonal arrays with mixed levels. J. Statist. Plann. Inference 56 295-306.

Suen, C., Chen, H. and Wu, C. F. J. (1997). Some identities on $q^{n-m}$ designs with application to minimum aberration designs. Ann. Statist. 25 1176-1188.

TANG, B. and DENG, L. Y. (1999). Minimum $G_{2}$-aberration for non-regular fractional factorial designs. Ann. Statist. 27 1914-1926.

TANG, B. and WU, C. F. J. (1996). Characterization of minimum aberration $2^{n-m}$ designs in terms of their complementary designs. Ann. Statist. 24 2549-2559.

VAN LinT, J. H. (1999). Introduction to Coding Theory, 3rd ed. Springer, New York.

WANG, J. C. and WU, C. F. J. (1995). A hidden projection property of Plackett-Burman and related designs. Statist. Sinica 5 235-250.

Wu, C. F. J. and Hamada, M. S. (2000). Experiments: Planning, Analysis and Parameter Design Optimization. Wiley, New York.

Wu, C. F. J. and ZHANG, R. (1993). Minimum aberration designs with two-level and four-level factors. Biometrika 80 203-209.

DePaRTMENT OF STATISTICS

UNIVERSITY OF MICHIGAN

ANN ARBOR, MichigAN 48109-1285

E-MAIL: jeffwu@umich.edu hqxu@umich.edu 